\title{
Cyanolichen microbiome contains novel viruses that encode genes to promote microbial metabolism
}

\author{
Alise J. Ponsero (iD $^{1,2}$, Bonnie L. Hurwitz ${ }^{1}$, Nicolas Magain (iD ${ }^{3,4}$, Jolanta Miadlikowska ${ }^{3}$, François Lutzoni ${ }^{3}$ and Jana M. U'Ren (iD ${ }^{1 凶}$
}

(c) The Author(s) 2021

Lichen thalli are formed through the symbiotic association of a filamentous fungus and photosynthetic green alga and/or cyanobacterium. Recent studies have revealed lichens also host highly diverse communities of secondary fungal and bacterial symbionts, yet few studies have examined the viral component within these complex symbioses. Here, we describe viral biodiversity and functions in cyanolichens collected from across North America and Europe. As current machine-learning viraldetection tools are not trained on complex eukaryotic metagenomes, we first developed efficient methods to remove eukaryotic reads prior to viral detection and a custom pipeline to validate viral contigs predicted with three machine-learning methods. Our resulting high-quality viral data illustrate that every cyanolichen thallus contains diverse viruses that are distinct from viruses in other terrestrial ecosystems. In addition to cyanobacteria, predicted viral hosts include other lichen-associated bacterial lineages and algae, although a large fraction of viral contigs had no host prediction. Functional annotation of cyanolichen viral sequences predicts numerous viral-encoded auxiliary metabolic genes (AMGs) involved in amino acid, nucleotide, and carbohydrate metabolism, including AMGs for secondary metabolism (antibiotics and antimicrobials) and fatty acid biosynthesis. Overall, the diversity of cyanolichen AMGs suggests that viruses may alter microbial interactions within these complex symbiotic assemblages.

ISME Communications(2021)1:56; https://doi.org/10.1038/s43705-021-00060-w

Lichens-defined as the symbiotic association between a filamentous fungus (mycobiont) and at least one photosynthetic organism (photobiont) - grow on a broad array of substrates in terrestrial, freshwater, and marine intertidal ecosystems from the poles to the tropics [1]. The vast majority of lichens contain green alga (Chlorophyta) as their main photobiont, but $>1500$ species of lichen-forming fungi have Nostoc cyanobacteria either as primary photobionts (forming bi-membered lichens) or as secondary photobionts (forming tri-membered lichens with a green alga as the primary photobiont) [2]. In exchange for photosynthate (and fixed nitrogen from Nostoc cyanobiont), the fungal partner provides the photobiont with carbon dioxide, inorganic ions, and protection from light [2]. More recently, molecular studies have shown that lichens also contain cryptic secondary bacterial and fungal symbionts [3-5], yet few studies to date have examined viruses that associate with these complex microbial communities (but see [6]).

Viruses infect all domains of life and are the most abundant biological entity on Earth [7]. Previous studies have shown that viruses that infect bacteria are evolutionarily tuned to their hosts in a given environment toward two main goals: (i) promotion of viral replication for lysis or (ii) co-existence within the host genome as a prophage [8]. To do so, viruses can encode host genes (i.e., auxiliary metabolic genes (AMGs; [9]) that promote viral replication through manipulation of the host's metabolism. For example, marine cyanophages encode $p s b A$ to increase host photosynthesis and drive replication when host $p s b A$ is inhibited by high light [10]. Viral AMGs can provide important clues into host adaptation, metabolic bottlenecks, key ecosystem functions, and interactions among members of a microbial community [8].

Viral sequences in metagenomes can be detected using reference-based methods, but these methods often are hampered by the limited diversity of viral genomes in reference databases (see [11]). Thus, an emerging approach is to apply machinelearning (ML) algorithms that use composition-based pattern detection. ML models identify a set of features that signal a viral origin (e.g., relative synonymous codon usage, gene density, strand shifts, number of hits to the Prokaryotic Virus Orthologous Groups (pVOGs) database), thus generalizing the identification of all viral sequences and enabling better detection of novel viruses [11-13]. These new approaches provide exciting avenues for detecting novel viral sequences in metagenomes and are key to investigating complex microbial symbioses such as lichens.

Here, we explored viral biodiversity in 11 cyanolichen metagenomes (Supplementary Table S1) representing nine species of the genus Peltigera sampled from North America, Finland, Iceland, and Panama, and one species from the sister genus Solorina (Fig. 1a) [14]. We predicted viruses with three ML tools (MARVEL, Vibrant, VirSorter) [11-13]. However, as repetitive regions of eukaryotic genomes can be falsely identified as viral [15], we first tested the false positive rate for each $\mathrm{ML}$ tools using a mock lichen community (Supplementary Table S2; Supplementary Fig. S1)

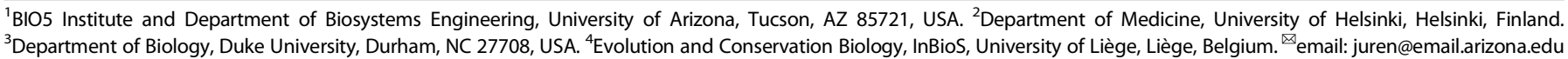

Received: 19 June 2021 Revised: 23 September 2021 Accepted: 27 September 2021

Published online: 15 October 2021 
a

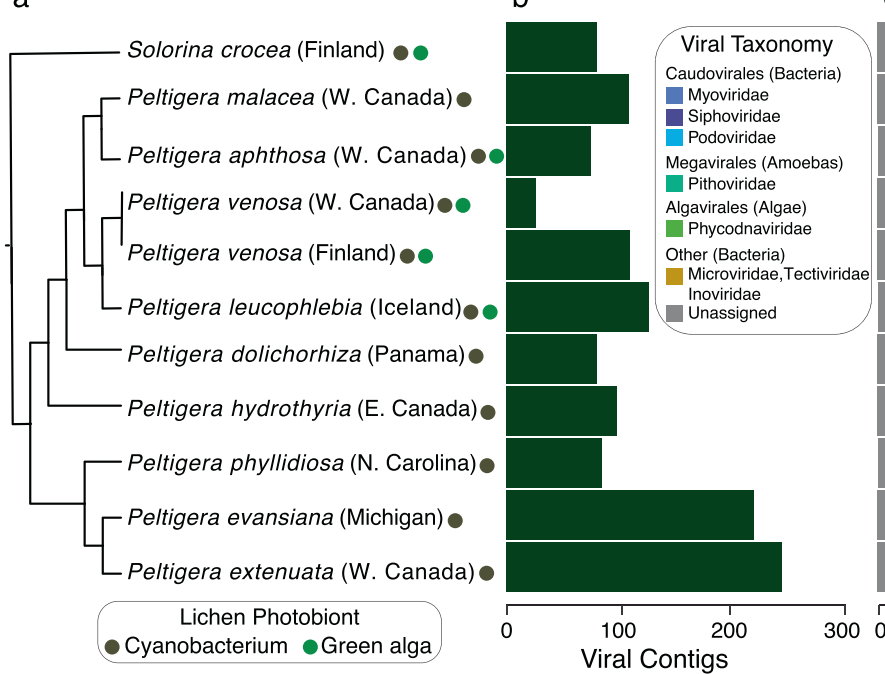

C

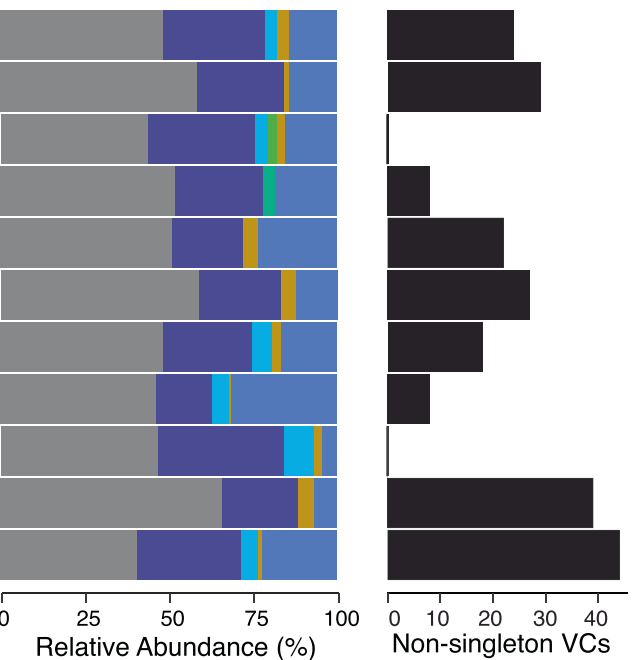

Fig. 1 Cyanolichens harbor diverse viral communities that infect prokaryotic and eukaryotic hosts in lichen thalli. a Schematic tree illustrating the phylogenetic relationships among lichen mycobionts (Peltigerales) (see Supplementary Materials). Colored circles indicate the photobiont composition of each lichen (see legend). Names associated with two colored circles are tri-membered lichens, whereas names associated with one colored circle are bi-membered cyanolichens (see Supplementary Table S1 for photobiont details). b Bar graph of the number of cyanolichen viral contigs predicted per sample, which accounted for $0.6-3.4 \%$ of the total reads per sample. c Taxonomic classification of viral contigs identified 15 viral families that infect prokaryotic ( $n=802$ contigs) and eukaryotic hosts ( $n=28$ contigs), although $471(36.2 \%)$ contigs could not be classified (Supplementary Table S3). d Distribution of non-singleton viral clusters (VCs) among samples. Among the 133 VCs, 50 were unique to a cyanolichen sample.

a

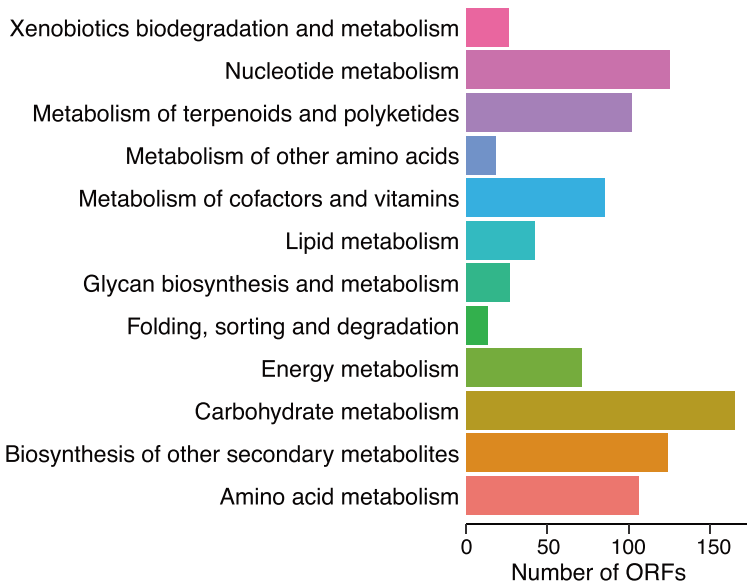

b

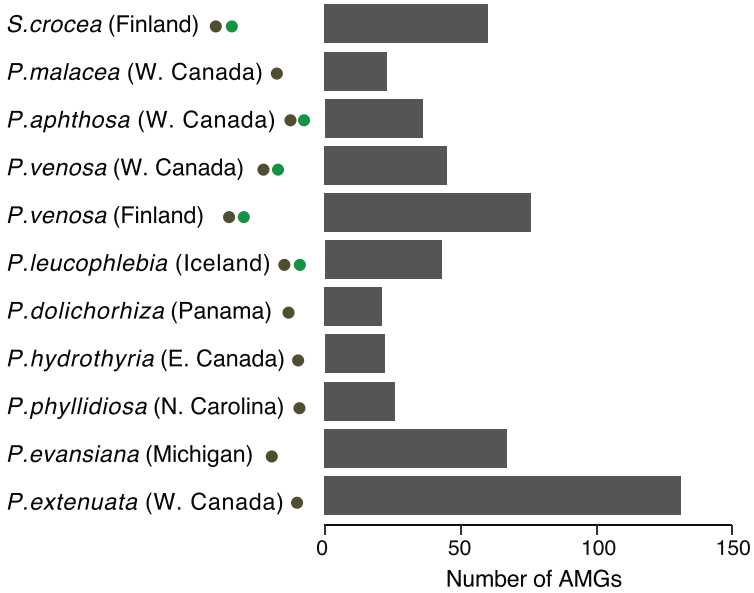

Fig. 2 Cyanolichen viruses encode diverse auxiliary metabolic genes (AMGs) for amino acid, nucleotide, carbohydrate, and secondary metabolite metabolism. a Number of AMGs per KEGG pathway. In total, AMGs were found on 249 contigs, including 9 of the 27 circular viral genomes. Most putative cyanolichen viral contigs contained one or two ORFs with KEGG annotation (Supplementary Table S4). b Number of AMGs per cyanolichen sample.

and then developed a pipeline to remove eukaryotic sequences prior to viral detection and apply a stringent cutoff to limit false positives (i.e., minimum of $10 \%$ of open reading frames (ORFs)/contig hit a previously identified viral protein) (Supplementary Fig. S2).

Every cyanolichen thallus contained putative viral sequences (range 27-254; Fig. 1b), although we observed no pattern between viral abundance and thallus type (bi- or tri-membered) or geographic location (Fig. 1b). In total, we predicted 1301 nonredundant viral contigs with high confidence across all metagenomes (Supplementary Fig. S3; Supplementary Table S3), including 116 predicted prophage sequences and 27 complete viral genomes that represent $8.9 \%$ and $2.0 \%$ of total contigs, respectively. The majority of viral contigs were classified as bacteriophages from the Caudovirales (61.2\%) (Fig. 1c). Twentyeight contigs also matched nine viral families that infect eukaryotes, including viruses of Phycodnaviridae that infect green algae [16] (Fig. 1c). Our taxonomic results are consistent with the observed bacterial communities in these samples (dominated by Proteobacteria and Cyanobacteria; Supplementary Fig. S4a), as well as our in silico predictions of viral hosts (Supplementary Fig. S4b). Additional eukaryotic viruses likely associate with algal photobionts of tri-membered lichens (see Fig. 1a) and fungal mycobionts in lichen thalli [6], but biases in both computational 
methods and databases towards phages may have limited their detection and classification (e.g., 36.2\% of viruses were unclassified; Fig. 1c; Supplementary Table S3). Additionally, many algal and fungal viruses are dsRNA viruses that cannot be detected with metagenomic data $[6,17]$.

To assess the novelty of cyanolichen viruses, we compared our contigs to $>400000$ previously published terrestrial viral contigs and genomes. The majority of our contigs $(n=966)$ did not form viral clusters (VCs) with non-lichen sequences and were classified as singletons or outlier VCs in network analysis (Supplementary Fig. S5). The remaining 335 contigs formed 133 VCs across the 11 metagenomes (Fig. 1d), with limited overlap to previously published sequences (i.e., only 28 VCs contained cyanolichen viral contigs and IMG/RefSeq sequences) (Supplementary Fig. S5) or among different cyanolichen samples (Supplementary Fig. S6). Similarly, we observed little functional overlap among our contigs (i.e., $96 \%$ of cyanolichen viral protein clusters (PCs) were singletons), consistent with high viral functional diversity [7]. Increasing sequencing depth would likely recover additional viruses and greater overlap with other ecosystems and among samples, but the lack of VC overlap also may reflect novel viral diversity and high geographic turnover of non-cyanobacterial lichen-associated bacterial communities [3] similar to turnover of marine viral communities according to host identity and physical or chemical properties of the environment $[8,18]$.

Consistent with the ability of phages in other ecosystems to encode host genes to drive host metabolism [8, 9], 550 of 21855 predicted ORFs had significant matches to metabolic KEGG HMM profiles (Fig. 2; Supplementary Table S4). Although putative AMGs represent a small fraction of total ORFs, they occurred on $19 \%$ of contigs (249 contigs with 1-2 AMGs per contig; see Supplementary Tables S4 and S5) and included diverse KEGG pathways such as amino acid, nucleotide, and carbohydrate metabolism (Fig. 2; Supplementary Tables S6 and S7). One of the most abundant AMGs in cyanolichens was the $r f b$ operon (21 contigs from 8 metagenomes), which is involved in the production of lipopolysaccharides and extracellular polymeric substances (EPS) in gram-negative bacteria [19] (Supplementary Table S7). The high number of contigs carrying the complete operon is consistent with the potential ecological importance of EPS for lichen thalli formation, water retention, and microbial communication (reviewed by Spribille et al. [20]). Putative AMGs also included KEGG pathways for secondary metabolism, although contigs did not contain complete secondary metabolite gene clusters.

In conclusion, numerous tools and approaches have been used to identify viruses in marine, human-associated, and soil metagenomes, yet viral detection remains challenging in complex and under-explored eukaryotic host-associated metagenomes. Here, we illustrate the diversity, novelty, and functional potential of viruses in cyanolichens and identify AMGs for metabolic pathways not previously described in viruses in other ecosystems (Supplementary Table S7). The diversity of viral AMGs in cyanolichens, including numerous AMGs for secondary metabolism, suggests viruses may modify interactions among complex microbial partners within lichens. Although viral novelty, microbial complexity, and inability to easily culture hosts in cyanolichen metagenomes limited our ability to link AMGs to specific hosts, future work will seek to decipher viral-host associations and the functional roles of AMGs in the lichen symbiosis using single viral particle sequencing.

\section{DATA AVAILABILITY}

Data used in this study are available at the National Center for Biotechnology Information (NCBI) Sequence Read Archive (SRA) (see Supplementary Table S1 for accession numbers). Predicted viral reads and AMGs are available on figshare (https://doi.org/10.6084/m9.figshare.c.5444376.v1). All code is available on GitHub (https://github.com/aponsero/) including the complete pipeline for (i) identifying viral contigs using VirSorter, Vibrant, and MARVEL (Viral_hunt_snakemake); (ii) validating that contigs are of viral origin (Viral_confirmation_snakemake); and (iii) code to run VirFinder in parallel on a High-Performance Computer (HPC) (VirFinder_parrallel_eval).

\section{REFERENCES}

1. Lutzoni F, Miadlikowska J. Lichens. Current Biol. 2009;19:R502-R503.

2. Nash TH. Lichen Biology. Cambridge, UK: Cambridge University Press; 1996.

3. Hodkinson BP, Gottel NR, Schadt CW, Lutzoni F. Photoautotrophic symbiont and geography are major factors affecting highly structured and diverse bacterial communities in the lichen microbiome. Environ Microbiol. 2012;14:147-61.

4. U'Ren JM, Lutzoni F, Miadlikowska J, Zimmerman NB, Carbone I, May G, et al. Host availability drives distributions of fungal endophytes in the imperiled boreal realm. Nat Ecol Evol. 2019;3:1430-7.

5. Arnold AE, Miadlikowska J, Higgins KL, Sarvate SD, Gugger P, Way A, et al. A phylogenetic estimation of trophic transition networks for ascomycetous fungi: are lichens cradles of symbiotrophic fungal diversification? Syst Biol. 2009;58: 283-97.

6. Petrzik K, Vondrák J, Barták M, Peksa O, Kubešová O. Lichens-a new source or yet unknown host of herbaceous plant viruses? Eur J Plant Pathol. 2014;138: 549-59.

7. Paez-Espino D, Eloe-Fadrosh EA, Pavlopoulos GA, Thomas AD, Huntemann M, Mikhailova N, et al. Uncovering Earth's virome. Nature. 2016;536:425-30.

8. Hurwitz BL, Westveld AH, Brum JR, Sullivan MB. Modeling ecological drivers in marine viral communities using comparative metagenomics and network analyses. Proc Natl Acad Sci USA. 2014;111:10714-9.

9. Breitbart $M$, Thompson LR, Suttle CA, Sullivan MB. Exploring the vast diversity of marine viruses. Oceanography. 2007;20:135-9.

10. Mann NH, Cook A, Millard A, Bailey S, Clokie M. Marine ecosystems: bacterial photosynthesis genes in a virus. Nature. 2003;424:741.

11. Roux $S$, Enault $F$, Hurwitz BL, Sullivan MB. VirSorter: mining viral signal from microbial genomic data. PeerJ. 2015;3:e985.

12. Amgarten D, Braga LPP, da Silva AM, Setubal JC. MARVEL, a tool for prediction of bacteriophage sequences in metagenomic bins. Front Genet. 2018;9:304.

13. Kieft K, Zhou Z, Anantharaman K. VIBRANT: automated recovery, annotation and curation of microbial viruses, and evaluation of viral community function from genomic sequences. Microbiome. 2020;8:90.

14. Cornet L, Magain N, Baurain D, Lutzoni F. Exploring syntenic conservation across genomes for phylogenetic studies of organisms subjected to horizontal gene transfers: a case study with Cyanobacteria and cyanolichens. Mol Phylogenet Evol. 2021;162:107100.

15. Ponsero AJ, Hurwitz BL. The promises and pitfalls of machine learning for detecting viruses in aquatic metagenomes. Front Microbiol. 2019;10:806.

16. Wilson WH, Van Etten JL, Allen MJ. The Phycodnaviridae: the story of how tiny giants rule the world. Curr Top Microbiol Immunol. 2009;328:1-42.

17. Marzano SL, Nelson BD, Ajayi-Oyetunde O, Bradley CA, Hughes TJ, Hartman GL, et al. Identification of diverse mycoviruses through metatranscriptomics characterization of the viromes of five major fungal plant pathogens. J Virol. 2016;90:6846-63

18. Brum JR, Ignacio-Espinoza JC, Roux S, Doulcier G, Acinas SG, Alberti A, et al. Patterns and ecological drivers of ocean viral communities. Science. 2015;348: 1261498.

19. Tsukioka Y, Yamashita Y, Oho T, Nakano Y, Koga T. Biological function of the dTDP-rhamnose synthesis pathway in Streptococcus mutans. J Bacteriol. 1997; 179:1126-34.

20. Spribille T, Tagirdzhanova G, Goyette S, Tuovinen V, Case R, Zandberg WF, et al. 3D biofilms: in search of the polysaccharides holding together lichen symbioses. FEMS Microbiol Lett. 2020;367:fnaa023.

\section{ACKNOWLEDGEMENTS}

Funding for this work was provided by NSF [OCE-1639614 Planet Microbe to BLH DEB-1556995 and DEB-1541548 to FL and JM] and Gordon and Betty Moore Foundation [GBMF 8751 to BLH]. Lichen sampling was funded by NSF grants DEB1046065 and DEB-1541548 to FL and JM.

\section{AUTHOR CONTRIBUTIONS}

Designed research: JMU, BLH, AJP; Performed research: AJP, JMU; Contributed data or analytic tools: NM, FL, JM; Analyzed data: AJP, JMU, BLH; Wrote the paper: AJP, JMU, $B L H$, with contributions from all authors. 


\section{COMPETING INTERESTS}

The authors declare no competing interests.

\section{ADDITIONAL INFORMATION}

Supplementary information The online version contains supplementary material available at https://doi.org/10.1038/s43705-021-00060-w.

Correspondence and requests for materials should be addressed to Jana M. U'Ren.

Reprints and permission information is available at http://www.nature.com/ reprints

Publisher's note Springer Nature remains neutral with regard to jurisdictional claims in published maps and institutional affiliations.
Open Access This article is licensed under a Creative Commons Attribution 4.0 International License, which permits use, sharing, adaptation, distribution and reproduction in any medium or format, as long as you give appropriate credit to the original author(s) and the source, provide a link to the Creative Commons license, and indicate if changes were made. The images or other third party material in this article are included in the article's Creative Commons license, unless indicated otherwise in a credit line to the material. If material is not included in the article's Creative Commons license and your intended use is not permitted by statutory regulation or exceeds the permitted use, you will need to obtain permission directly from the copyright holder. To view a copy of this license, visit http://creativecommons. org/licenses/by/4.0/.

(c) The Author(s) 2021 